\title{
Dimensioning Optical Networks under Traffic Growth Models
}

\author{
Tapan Kumar Nayak \\ Dept of Electrical Communication Engineering \\ Indian Institute of Science \\ Bangalore, India 560012
}

\author{
Kumar N. Sivarajan \\ Tejas Networks \\ Khanija Bhavan, 49, Race Course Road \\ Bangalore, india 560001
}

\begin{abstract}
In this paper we introduce a traffic growth model for optical networks with time-varying traffic arrivals assuming the nodes are capable of full wavelength conversion. We propose a network dimensioning method based on the traffic growth model and it eventually results in a nonlinear optimization problem with cost minimization as the objective and route absorption probabilities as the constraints. The absorption probabilities can be obtained from transient analysis of a Markov chain. Computation of exact absorption probabilities requires huge computing resources and is thus feasible only for small networks. We consider a reduced load approximation for estimating absorption probabilities of a wavelength routed network with arbitrary topology and traffic patterns. We show that the approximation method performs very well for optical networks with long-lived lightpaths.
\end{abstract}

\section{INTRODUCTION}

Wavelength-division multiplexing(WDM) [1] in optical networks is a promising technology to utilize the enormous bandwidth of optical fiber and it offers the capability of building very large wide-area networks with throughputs of the order of gigabits per sec for each node [2]. In this work we consider that all the network nodes have full wavelength conversion capability and it provides improved utilization of the available fiber bandwidth in a WDM network. Significant advances in optical and electronic device technologies have made wavelength conversion feasible. Optical wavelength converters are still in an experimental stage; however, with electronic wavelength crossconnects $(W X C)$, it is easier to realize wavelength conversion [3]. With full wavelength conversion capability, an optical network is equivalent to a conventional circuit switched network.

A network dimensioning method allocates appropriate capacities to the WDM links based on the network topology and traffic requirements. Many network dimensioning methods are based on mathematical models with cost minimization as the objective and grade-of-service constraints expressed in terms of end-to-end blocking probabilities[4]. The models turn out to be nonlinear optimization problems with a rather large number of variables and in some formulations many nonlinear constraints. A straightforward solution method is to use the algorithms for nonlinear constrained minimization [5], [6]. It involves the computation of blocking probabilities for different values of capacity on the links. For a large circuit-switched network, Kelly proposed an efficient method to estimate the loss probabilities based on solving a fixed point problem involving Erlang's formula under the assumption of independent blocking and showed that under a limiting regime the estimates of loss probabilities converge to the correct values [7]. Several models are considered in the literature to approximate the blocking probabilities in all-optical networks with and without wavelength changers [8]-[11]. In all these cases, the dimensioning problem is solved assuming the network is in a steady state condition.

Traditional networking technologies were designed for a world of moderate traffic growth with relatively stable and predictable traffic patterns. Such systems were simply not designed to handle the explosive growth in bandwidth demand coupled with the dynamic nature of traffic patterns created by emerging broadband and Internet applications. So application of the blocking model is not appropriate because the traffic will not reach a steady state as it is growing constantly and unpredictably. Such a dramatic shift in the environment requires a fundamental shift in network architectures in order to realize the full potential of modern applications.

In this paper we consider a traffic growth model and use the concept of absorption probability instead of blocking probability to express the grade of service of a network. The dimensioning problem based on absorption probability is solved for constant rates of traffic arrivals and the details of the analytical model can be found in [12]. Here we consider the more general case of time-varying traffic arrivals, which is a better assumption and more relevant in the current scenario of rapidly growing traffic in optical networks.

We elaborate on this traffic model in the next section.

\section{TRAFFIC MODEL AND OVERVIEW}

Lightpaths are assumed to arrive randomly according to a statistical model and have to be set up on the optical layer. Some lightpaths may depart as well but it is assumed that, on average, the number of lightpaths will keep increasing and eventually we will have to reject a lightpath request. Thus the rate of arrival of lightpath requests exceeds the rate of termination of lightpaths, and the network is not in equilibrium. This is a reasonable model today since the lightpaths are long-lived and the traffic in the network is growing. We are interested in dimensioning the WDM links so that the first lightpath request rejection will occur, with high probability, after a specified period of time, $T$. The longevity, combined with the cost of a high bandwidth lightpath today, means that a network operator is unlikely to reject a lightpath request. Rather, he would like to upgrade his network by the addition of more capacity on existing links in order to accommodate the lightpath request. The time period $T$ corresponds approximately to the time by which the operator must institute such upgrades in order to avoid rejecting lightpath requests. So if a network has an absorption probability of 
$p$ at time $T$, it means the operator has to add additional capacity with probability $p$ by time $T$ in order to be able to set up all the requested lightpaths/connections.

However, the dimensioning problem is solved based on a nonlinear optimization model and it involves the computation of absorption probabilities for a given set of capacities on the WDM links. The network can be modeled by a Markov chain where a state is represented by the set of lightpaths in progress. Estimation of absorption probability is based on the transient analysis of the Markov chain. In this paper we introduce an efficient method to compute the absorption probabilities which shows excellent performance even for large networks.

The rest of the paper is organized as follows. In Section III we compute the exact absorption probabilities for single and double link networks. In Section IV, we consider a large network, outline the traffic assumptions and describe the method for calculation of approximate absorption probabilities. In Section $\mathrm{V}$, we formulate the optimization problem to determine the link capacities. We present our results in Section VI and conclude in Section VII.

\section{EXACT SOLUTION FOR Single AND DOUblE LINK NETWORKS}

In our proposed reduced load approximation method, the approximate absorption probability of a route in a large network is calculated based upon the individual link absorption probabilities with the assumption that the links are independent but the arrival rates are thinned. The details of the method are discussed in section IV. This motivates us to study a single link network in details.

Here we consider a single link network of capacity $K$. The busy wavelength distribution can be modeled by a birth-death process where a state of the Markov chain represents the number of lightpaths in progress. We assume the arrivals are Poisson with $\lambda(t)$ as the state-independent arrival rate at time $t$. Holding times are assumed exponential with constant mean $1 / \mu$. The birth-death model for a single link with the above assumptions is shown in Fig. 1.

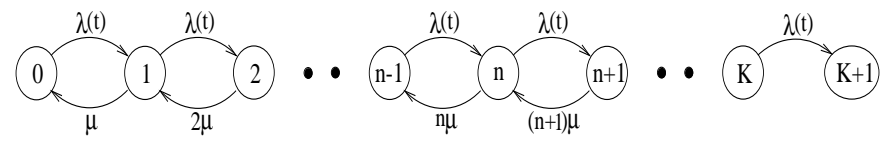

Fig. 1. Markov chain of the busy wavelength distribution on a single link

Let $P_{n}(t)$ be the probability of state $n$ at time $t$. So we can establish the following relations between the state probabilities [13]. The network is assumed to start with no lightpaths at all.

$$
\begin{aligned}
P_{n}^{\prime}(t)=- & (\lambda(t)+n \mu) P_{n}(t)+\lambda(t) P_{n-1}(t)+ \\
& (n+1) \mu P_{n+1}(t) \quad \text { for } 1 \leq n \leq K-1, \\
P_{0}^{\prime}(t)=-\lambda(t) P_{0}(t)+\mu P_{1}(t) & \\
P_{K}^{\prime}(t)= & (\lambda(t)+K \mu) P_{K}(t)+\lambda(t) P_{K-1}(t) \\
P_{K+1}^{\prime}(t)= & \lambda(t) P_{K}(t)
\end{aligned}
$$

Initial Condition : $P_{0}(0)=1, \quad P_{n}(0)=0$ for $n \geq 1$. Define

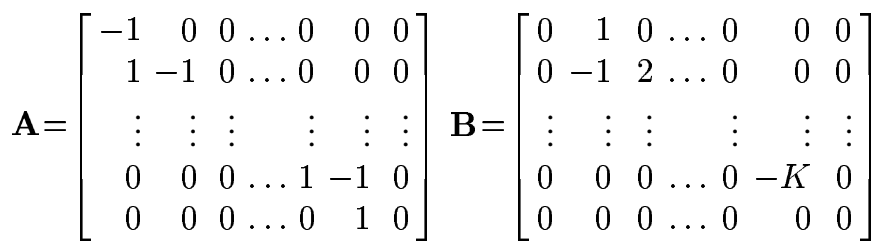

$$
\begin{aligned}
& \mathbf{P}(t)=\left[\begin{array}{llll}
P_{0}(t) P_{1}(t) \ldots P_{K+1}(t) & \ldots
\end{array}\right]^{T} \text { and } \mathbf{P}(0)=\left[\begin{array}{llll}
1 & 0 & \ldots & 0
\end{array}\right]^{T} .
\end{aligned}
$$

With these notations the system (1)-(4) can be written as

$$
\mathbf{P}^{\prime}(t)=(\lambda(t) \mathbf{A}+\mu \mathbf{B}) \mathbf{P}(t)=\mathbf{M}(t) \mathbf{P}(t),
$$

where $\mathbf{M}(\mathrm{t})$ is a $(K+2) \times(K+2)$ matrix of variable coefficients.

We assume the arrival rate $\lambda(t)$ as a continuous function of time $t$. Then the system of linear differential equations (5) with the given initial values $\mathbf{P}(0)$, possesses a unique continuous solution on the interval $t \geq 0$. ([14], p. 20)

Again it can be proved that the elements of the solution vector are bounded by 0 and 1 and the sum is 1 for all $t \geq 0$. Hence, a solution exists and it is unique for all $t \geq 0$. The explicit solution of (5) is complicated. So an alternative is to compute the solution through numerical techniques.

The link will be absorbed if all the $K$ wavelengths are busy and another arrival occurs. So the $(K+1)$-th state is an absorbing state from which no exit is possible. From (4) it follows that $P_{K+1}^{\prime}(t) \geq 0$ as $\lambda(t) \geq 0$ and $0 \leq P_{K}(t) \leq 1$ for all $t$. So, $P_{K+1}(t)$ is a monotonically non-decreasing function of $t$ with $P_{K+1}(0)=0$. The explicit solution of the absorption probability $P_{K+1}(t)$ is complicated. But it can be computed easily by numerical methods [15].

For a double link network, we use a three-dimensional Markov chain model to calculate the exact absorption probability. We do not describe the analytical details of the 3-D Markov chain model here; they can be found in [12]. Although the explicit solution is unknown, the exact absorption probability can be computed by numerical methods [15].

\section{Approximate Solution for General Networks}

We now describe an approximation method to calculate the absorption probabilities for a general optical network with the following assumptions.

\section{A. Model Assumptions}

1) The network consists of $J$ links connected in an arbitrary topology. Denote $C_{j}$ for the capacity in link $j, j=$ $1,2, \cdots, J$.

2) A route $r$ is a subset of links from $\{1,2, \cdots, J\}$. A lightpath in route $r$ uses $A_{j r}$ wavelengths from link $j$, $A_{j r} \in\{0,1\}$. Denote $\mathbf{R}$ for the set of routes.

3) Lightpaths requests arrive on route $r$ according to a timevarying Poisson process with rate $\lambda_{r}(t)$ at time $t$. 
4) Lightpath holding times are exponentially distributed with unit means and independent of earlier arrivals.

5) A link $j$ is absorbed at time $t$ if a lightpath requesting route $r$ arrives at time $t$ but there is no wavelength free in link $j$ provided $A_{j r}=1$.

Let us define the vectors $\mathbf{C}=\left(C_{j}, j=1,2, \cdots, J\right)$ and $\mathbf{n}=\left(n_{r}, r \in \mathbf{R}\right)$ when $n_{r}$ be the number of lightpaths in progress in route $r$. Now considering a $|\mathbf{R}|$-dimensional state space with a constraint $\mathbf{A n} \leq \mathbf{C}$, an explicit form might be thought to provide the complete solution of absorption probabilities. However, the number of routes and thus the size of the state space grows rapidly with the number of links. The size of state space also increases with the capacities of the links $C_{1}, C_{2}, \cdots, C_{J}$. So, it is impractical to find the explicit forms of absorption probabilities except for some small networks.

The assumption that all the links are independent with the sum of arrival rates $\sum_{r \in \mathbf{R}} A_{j r} \lambda_{r}(t)$ as the arrival rate on link $j$ will yield an upper bound on absorption probability. The absorption probability of the $j$-th link at time $t$ is

$$
P_{a b s}^{j}(t) \leq F\left(\sum_{r \in \mathbf{R}} A_{j r} \lambda_{r}(t), C_{j}\right), \quad j=1,2, \cdots, J,
$$

where $F(\lambda(t), K)$ is the absorption probability at time $t$ for a single link of capacity $K$ with time-dependent arrival rate $\lambda(t)$ and mean holding time unity. It can be computed from (5) as $P_{K+1}(t)$. But this may lead to a very conservative upper bound. As an alternative, the links can still be assumed independent if the arrival rates are thinned properly for each link and this results a good approximation of the absorption probabilities.

Consider a scalar function $g(p)$, so that $0 \leq g(p) \leq 1$ as $p$ varies from 0 to 1 . Now approximate the carried traffic on link $j$ at time $t$ by a Poisson process with arrival rate $\sum_{r} A_{j r} \lambda_{r}(t) \prod_{i \neq j}\left[g\left(P_{a b s}^{i}(t)\right)\right]^{A_{i r}}$ where $P_{a b s}^{i}(t)$ is the absorption probability of link $i$ at time $t$. It is assumed that when $A_{j r}=1$ the traffic of rate $\lambda_{r}(t)$ is thinned by a factor $g\left(P_{a b s}^{i}(t)\right)$ on each link $i \in r-\{j\}$ before being offered to link $j$ and the thinnings are independent from link to link and over all routes containing the link $j$. Now the success of the approximation method depends on the proper choice of the function $g(p)$. Motivated by the Erlang fixed point approximation in steady state condition [7], we consider $g(p)=1-p$ as a good choice. Apart from this, we can come up with different possible choices for $g(p)$ to obtain a better approximation.

Now the approximate absorption probability of link $j, j=$ $1,2, \cdots, J$ can be calculated from the following system of timedependent non-linear equations

$$
P_{a b s}^{j}(t)=F\left(\sum_{r \in \mathbf{R}} A_{j r} \lambda_{r}(t) \prod_{i \in r-\{j\}} g\left(P_{a b s}^{i}(t)\right), C_{j}\right) .
$$

So the absorption probability of a route $r \in \mathbf{R}$ at time $t$,

$$
L_{r}(t)=1-\prod_{j \in r}\left(1-P_{a b s}^{j}(t)\right) .
$$

Now we discuss briefly the method of solution for the system of eqns. (6). Consider each link $j, j=1,2, \ldots, J$ as an independent single link with arrival rate at time $t$, $\lambda^{j}(t)=\sum_{r \in \mathbf{R}} A_{j r} \lambda_{r}(t) \prod_{i \in r-\{j\}} g\left(P_{C_{i}+1}^{i}(t)\right)$ and generate the $\left(C_{j}+2\right)$ differential equations as shown in (1)-(4) for a single link network. So we have $J$ sets of homogeneous differential equations which are non-linear and coupled by the factor $\lambda^{j}(t)$. Now we combine all the equations in a single system. The total number of equations $N=\sum_{j=1}^{J} C_{j}+2 J$.

Define a probability vector $\mathbf{P}(t)$ of dimension $N$ so that $P_{n}^{j}(t)$ be the $\left(\sum_{i=1}^{j-1}\left(C_{i}+2\right)+n+1\right)$-th element of $\mathbf{P}(t)$.

Now combining the differential equations for all the links,

$$
\mathbf{P}^{\prime}(t)=(\mathbf{D}(t, \mathbf{P}) \mathbf{L}+\mathbf{U}) \mathbf{P}(t)=\mathbf{M}(t, \mathbf{P}) \mathbf{P}(t),
$$

where $\mathbf{L}$ and $\mathbf{U}$ are constant $N \times N$ lower and upper bidiagonal matrices respectively. $\mathbf{D}$ is a $N \times N$ diagonal matrix which is a function of $t$ and $\mathbf{P}$. Note that $\lambda^{j}(t)$ is the $\left(\sum_{i=1}^{j-1}\left(C_{i}+2\right)+n+\right.$ 1)-th diagonal element of $\mathbf{D}$ for all $j, j=1,2, \ldots, J$ and for all $n, n=0,1, \ldots, C_{j}+1$.

For the system of eqns. (8), it is not possible to obtain the explicit form of the solution in general but it can be proved that a probability solution exists and it is unique for all values of $t \geq 0$ provided $\lambda_{r}(t)$ is a continuous function of $t$ for all $r \in \mathbf{R}$. Although the analytical solution is unknown, the system (6) with the given initial conditions can be solved by numerical methods using ODE solvers [15].

\section{CAPACity Allocation}

Given the network topology and the cost to set up capacities on each link, we want to select the capacities so as to meet the absorption probability constraints while minimizing costs.

So the problem is to choose the capacity $C_{j}$ for each link $j$, $j=1,2, \ldots, J$, so as to minimize the linear cost $\sum_{j=1}^{J} Q_{j} C_{j}$ where $Q_{j}$ is a known positive price to set up unit capacity in link $j$, subject to the constraints that the absorption probability of any route $r \in \mathbf{R}$ at a given time $t=T$ should not exceed a given constant $P_{t h}$.

We are now ready to state the problem formally as mathematical program.

Objective function

$$
\min \sum_{j=1}^{J} Q_{j} C_{j}
$$

subject to

$$
\begin{aligned}
P_{a b s}^{j}(T) & =F\left(\sum_{r \in \mathbf{R}} A_{j r} \lambda_{r}(T) \prod_{i \in r-\{j\}} g\left(P_{a b s}^{i}(T)\right), C_{j}\right) \forall j, \\
L_{r}(T) & =1-\prod_{j \in r}\left(1-P_{a b s}^{j}(T)\right) \leq P_{t h}, \text { for all } r \in \mathbf{R}, \\
0 & \leq P_{a b s}^{j}(T) \leq 1, \quad C_{j} \in \mathbb{N}^{+} \quad \text { for all } j,
\end{aligned}
$$

where $\mathbb{N}^{+}$is a finite set of positive integers. 
This is an example of mixed integer nonlinear program or MINLP. Although some efficient algorithms are known for solving NLPs, no efficient algorithms are known for the solution of arbitrary MINLPs and it is extremely hard. We develop a heuristic to find a good solution using the techniques of $N L P$-relaxation and rounding. Note that if we relax $C_{j}$ to real numbers, the MINLP is converted to an NLP. Estimate the absorption probability for a non-integer capacity based on the absorption probabilities for integer capacities. Now round the NLP solution to integers to obtain the link capacities. Rounding up will always give a solution, but the performance can be improved by the following technique. Set a threshold between 0 and 1 for the fractional parts of the NLP solution. Round up to 1 if the fractional part is greater than the threshold value else round down to 0. Apply this method to all the reals of the NLP solution and calculate the absorption probabilities. If these are not acceptable on some links, adjust the threshold and recalculate the absorption probabilities.

\section{RESUlts AND DISCUSSION}

Here we present the numerical results of the exact absorption probabilities for a single link and the exact and approximate absorption probabilities for a double link as functions of time. We also consider a moderate-sized network ARPANET as an arbitrary network and solve the dimensioning problem for a given function of traffic arrival.

For our experiments, we consider a linear function to represent the traffic growth. For the the single link network, the arrival rate at time $t$,

$$
\lambda(t)=\lambda_{0}(1+t / \tau)
$$

where $\lambda_{0}$ is the initial arrival rate at time $t=0$ and $\tau$ is a constant parameter.

The exact absorption probabilities of a single link network of capacity 32 are calculated for different values of $\tau$ with $\lambda_{0}=14$ and the probabilities are plotted as functions of time in Fig. 2. It shows that the absorption probability is monotonically increasing with $t$ but decreasing with $\tau$.

For a two-link network, the upper bound, the exact absorption probability and the approximate absorption probabilities with 3 different choices of $g(p)$ are calculated as functions of time and the results are shown in Fig. 3. The arrival rate on route $i$ at time $t, \lambda_{i}(t)=\lambda_{i 0}(1+t / \tau)$, where $\lambda_{i 0}$ is the initial arrival rate at $t=0, i=1,2,3$.

It is observed from Fig. 3 that the approximations with all the 3 functions are very close to the exact values at low absorption probabilities. But the approximations with $g(p)=1-p$ and $g(p)=1-2 p+2 p^{2}$ deviate widely from the exact values at large absorption probabilities. This behavior can be explained as follows. For large values of absorption probability, the thinning of type-3 lightpaths are excessive and the thinned terms at $t, \lambda_{3}(t) g\left(P_{a b s}^{1}(t)\right)$ and $\lambda_{3}(t) g\left(P_{a b s}^{2}(t)\right)$ are almost zero. This is equivalent to a 2-link network with only type-1 and type-2 lightpaths with arrival rates $\lambda_{1}(t)$ and $\lambda_{2}(t)$ respectively and

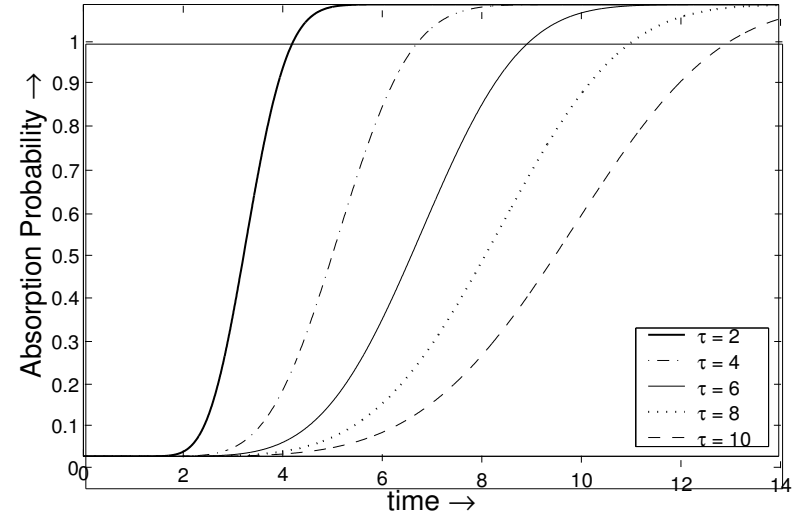

Fig. 2. The exact absorption probabilities of a single link network with capacity $K=32$, arrival rate at time $t, \lambda(t)=14(1+t / \tau)$ and mean holding time $1 / \mu=1$

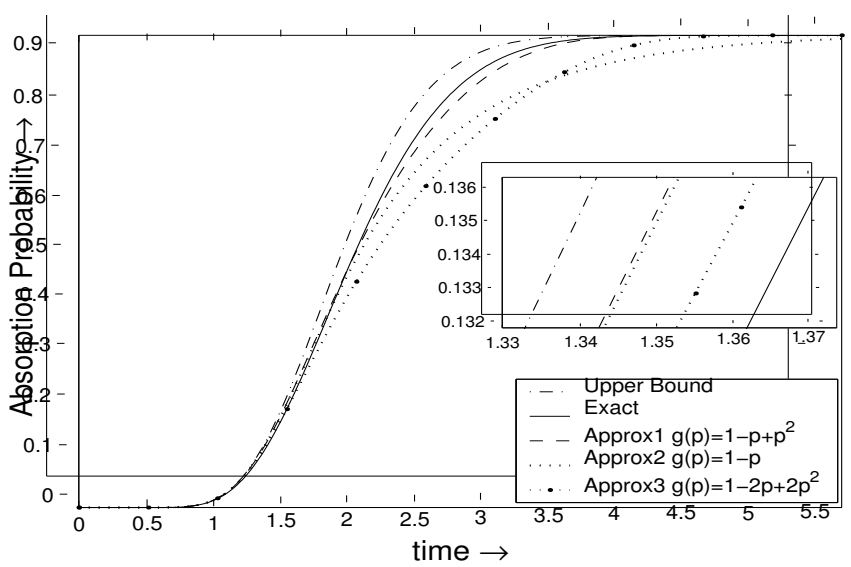

Fig. 3. Absorption probabilities of a two-link network with capacities $K_{1}=$ $K_{2}=16$, initial arrival rates $\lambda_{10}=\lambda_{20}=\lambda_{30}=6$, parameter $\tau=4$ and mean holding time $1 / \mu=1$. The inset figure is a zoom of the curves at low absorption probabilities.

the corresponding absorption probability is a significant under estimate of the exact absorption probability. The large underestimation at high absorption probabilities causes no problem in designing a network, in practice, because the required absorption probability is typically low ( 0.1 or less). Observe that the approximation with $g(p)=1-p+p^{2}$ is good for both low and high absorption probabilities.

We consider the ARPA network shown in Fig. 4 as an example of an arbitrary and moderately large topology to study the performance for arbitrary networks. Routes are selected as the shortest paths between any two nodes. The total number of possible routes is 190 considering only one route between any two nodes. Under shortest path routing, the maximum possible hop length is 6 and the number of routes with 1, 2, 3, 4, 5 and 6 hops are 32, 52, 56, 32, 17 and 1 respectively. Here we consider only the non-uniform traffic with $q=0.5$ where $\lambda_{H}(t)=q^{H-1} \cdot \lambda_{1}(t), \lambda_{H}(t)$ is the arrival rate on a $H$-hop route at time $t$ and $\lambda_{1}(t)=\lambda_{10}(1+t / 4), \lambda_{10}$ is the initial arrival rate at $t=0$ on a single hop route. 


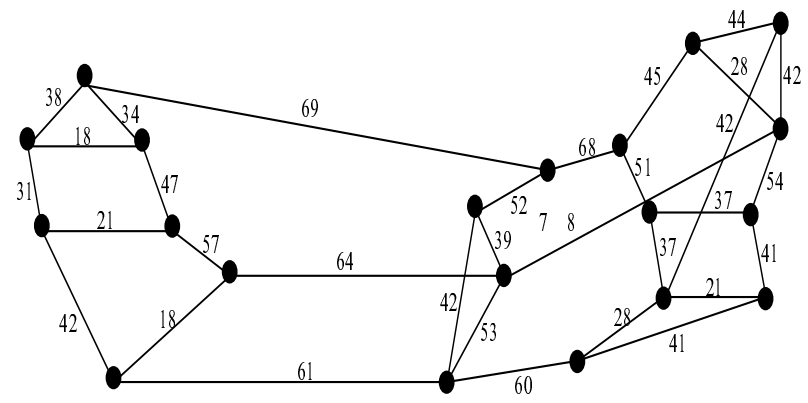

Fig. 4. A 20 node, 32 link network representing a skeleton of the original Arpanet. A set of capacities (column 4, Table I) is shown on different links of the network, as an example of a properly dimensioned network for a time period $T=2$ with $P_{t h}=0.1$.

We now solve the dimensioning problem so that the absorption probabilities of all the routes are less than $0.01(1 \%)$ and $0.1(10 \%)$ at time $T=1,23$ and 4 with $\lambda_{10}=6$ and the capacities allotted to various links of the networks are presented in Table I. We consider $g(p)=1-p$ because it is equivalent to $g(p)=1-p+p^{2}$ at low absorption probabilities but the complexity is less.

For an illustration, let us assume the mean holding time of a lightpath is 6 months. Allot the capacities based on the absorption probability as shown in Fig. 4. The absorption probabilities for all the routes are computed by the proposed reduced load approximation method which are all less than 0.1 and compared with the simulation at $T=2$ with $\lambda_{10}=6$. The simulated absorption probabilities are found slightly less than the approximate absorption probabilities in all the routes and the difference is more for larger hop routes. This ensures that the operator has a $90 \%$ or better chance of not having to upgrade the network for a time period of 1 year where the traffic arrival rate will be doubled every two years.

\section{CONCLUSION}

In this paper, we have introduced a traffic growth model for time-varying traffic in optical networks and proposed a dimensioning method based on a nonlinear optimization problem with cost minimization as the objective and route absorption probabilities as the constraints. Here we consider only fixed routing. In practice, when a certain route is saturated the operator is likely to use an alternative path to setup a new lightpath. The model described can be modified for alternate routing but this is a topic for further research.

\section{REFERENCES}

[1] C. A. Brackett, "Dense wavelength division multiplexing network principles and applications," IEEE Journal on Selected Areas in Communications, vol. 8, pp. 948-964, Aug. 1990.

[2] R. Ramaswami, "Multi-wavelength lightwave networks for computer communication," IEEE Communications Magazine, vol. 31, pp. 78-88, Feb 1993.

[3] R. Ramaswami and K. N. Sivarajan, "Optical Networks : A Practical Perspective," Morgan Kaufmann Publishers, San Fransisco, 1998.

[4] A Girard, "Routing and Dimensioning in circuit-switched networks," Addison-Wesley, 1990.

[5] D. Bertsekas. "Nonlinear Optimization," Athena Scientific, 1995
TABLE I

CAPACITIES ALLOTTED TO DIFFERENT LINKS OF THE ARPA NETWORK

\begin{tabular}{|c|c|c|c|c|c|c|c|c|}
\hline \multirow{3}{*}{$\begin{array}{l}\text { Link } \\
\text { No }\end{array}$} & \multicolumn{8}{|c|}{ Absorption Probability } \\
\hline & \multicolumn{2}{|c|}{$\mathrm{T}=1$} & \multicolumn{2}{|c|}{$\mathrm{T}=2$} & \multicolumn{2}{|c|}{$\mathrm{T}=3$} & \multicolumn{2}{|c|}{$T=4$} \\
\hline & 0.1 & 0.01 & 0.1 & 0.01 & 0.1 & 0.01 & 0.1 & 0.01 \\
\hline$\overline{1}$ & $\overline{21}$ & 25 & 31 & 36 & 39 & 44 & 45 & $\overline{50}$ \\
\hline 2 & 28 & 32 & 42 & 46 & 51 & 57 & 60 & 66 \\
\hline 3 & 14 & 17 & 21 & 25 & 26 & 30 & 30 & 35 \\
\hline 4 & 26 & 30 & 38 & 43 & 47 & 53 & 55 & 61 \\
\hline 5 & 12 & 16 & 18 & 22 & 23 & 27 & 27 & 31 \\
\hline 6 & 12 & 16 & 18 & 22 & 23 & 27 & 27 & 31 \\
\hline 7 & 40 & 45 & 61 & 67 & 75 & 82 & 88 & 95 \\
\hline 8 & 23 & 27 & 34 & 39 & 43 & 48 & 50 & 55 \\
\hline 9 & 46 & 51 & 69 & 75 & 85 & 93 & 100 & 108 \\
\hline 10 & 31 & 36 & 47 & 53 & 58 & 65 & 68 & 75 \\
\hline 11 & 38 & 42 & 57 & 62 & 70 & 77 & 82 & 89 \\
\hline 12 & $\overline{42}$ & 48 & 64 & 70 & 79 & 87 & 93 & 101 \\
\hline 13 & 28 & 32 & 42 & 48 & 53 & 59 & 61 & 68 \\
\hline 14 & 35 & 40 & 53 & 59 & 66 & 73 & 77 & 84 \\
\hline 15 & 40 & 45 & 60 & 67 & 74 & 81 & 87 & 95 \\
\hline 16 & 26 & 30 & 39 & 45 & 49 & 55 & 57 & 63 \\
\hline 17 & 34 & 40 & 52 & 58 & 65 & 72 & 76 & 83 \\
\hline 18 & 58 & 64 & 87 & 95 & 109 & 117 & 127 & 136 \\
\hline 19 & 45 & 51 & 68 & 75 & 85 & 92 & 99 & 107 \\
\hline 20 & 19 & 22 & 28 & 32 & 35 & 40 & 41 & 46 \\
\hline 21 & 27 & 32 & 41 & 47 & 51 & 57 & 60 & 66 \\
\hline 22 & 33 & 38 & 51 & 57 & 63 & 69 & 73 & 81 \\
\hline 23 & 30 & 35 & 45 & 51 & 56 & 62 & 66 & 72 \\
\hline 24 & 25 & 29 & 37 & $\overline{42}$ & 46 & 52 & 54 & 60 \\
\hline 25 & 14 & 17 & 21 & 25 & 26 & 30 & 30 & 35 \\
\hline 26 & 28 & 33 & 42 & 48 & 53 & 59 & 62 & 68 \\
\hline 27 & 25 & 29 & 37 & 42 & 46 & 52 & 54 & 60 \\
\hline 28 & 28 & 32 & 41 & 46 & 51 & 57 & 60 & 66 \\
\hline 29 & 36 & 41 & 54 & 60 & 67 & 74 & 78 & 86 \\
\hline 30 & 19 & 22 & 28 & 32 & 35 & 40 & 40 & 46 \\
\hline 31 & 29 & 34 & 44 & 50 & 55 & 61 & 64 & 71 \\
\hline 32 & 28 & 32 & 42 & 47 & 52 & 57 & 60 & 67 \\
\hline$\overline{\overline{\text { Tot }}}$ & $\overline{\overline{940}}$ & $\overline{1083}$ & $\overline{1412}$ & $\overline{\overline{1586}}$ & $\overline{1756}$ & $\overline{1949}$ & $\overline{2051}$ & $\overline{2257}$ \\
\hline
\end{tabular}

[6] S. Nash and A. Sofer, "Linear and Nonlinear Programming," McGrawHill, New York, 1996.

[7] F. P. Kelly, "Blocking probabilities in large circuit-switched networks," Advances in Applied Probability, vol. 18, pp. 473-505, 1986.

[8] A. Birman, "Computing approximate blocking probabilities for a class of all-optical networks," IEEE Journal on Selected Areas in Communications, vol. 14, pp. 852-857, June 1996.

[9] A. Sridharan and K. N. Sivarajan, "Blocking in all-optical networks," Proceedings, IEEE INFOCOM 2000, March 2000.

[10] R. A. Barry and P. A. Humblet, "Models of blocking probability in alloptical networks with and without wavelength changers" IEEE Journal on Selected Areas in Communications, vol. 14, N0. 5, pp. 858-867, June 1996.

[11] T. Tripathi and K. N. Sivarajan, "Computing approximate blocking probabilities for wavelength-routed all-optical networks with limited-wavelength conversion," Proceedings, IEEE INFOCOM '99.

[12] T. K. Nayak and K. N. Sivarajan, "A new approach to dimensioning optical networks," IEEE Journal on Selected Areas in Communications, vol. 20, No. 1, January 2002.

[13] W. Feller, "An Introduction to Probability Theory and its Applications," vol. 1, 3rd edn. Wiley, New York, 1968.

[14] E. A. Coddington and N. Levinson, "Theory of Ordinary Differential Equations," McGraw-Hill, New York, 1955.

[15] S. C. Chapra and R. P. Canale, "Numerical Methods for Engineers, With Programming and Software Applications" 3rd edn. McGraw-Hill, New York, 1998. 